\title{
LA INTEGRACIÓN ESCOLAR DE ALUMNOS CON NECESIDADES EDUCATIVAS ESPECIALES ENTRE LA REALIDAD Y EL DESEO
}

\author{
Gerardo Echeita \\ I.E.S "María de Molina" de Madrid
}

\begin{abstract}
RESUMEN. En este trabajo se valora el desarrollo global del Programa de Integración Escolar de Alumnos con Necesidades Educativas Especiales, que impulsa el M.E.C., y se analizan algunos factores que, a juicio del autor, están condicionando negativamente su alcance. Entre estos se destacan los referidos a ciertas actitudes de complacencia con los logros alcanzados, los relativos a ciertas condiciones de diseño del programa y los que tienen que ver con la ausencia de una politica de desarrollo curricular.

ABSTRACT. In this paper the Integration Programme for Pupils wtih Special Educational Needs developped by the Ministry of Education and Culture is valued. Some factores involved in the perceived negative scope are analized, particulary regarding with social attitudes of satisfaction about present results, design conditions and lack of curriculum development policy.
\end{abstract}

\section{Introducción}

En unas recientes declaraciones con motivo de la presentación del informe del INCE sobre los resultados de la primera evaluación de la ESO, el Secretario General de Educación, Eugenio Nasarre, decía lo siguiente:

"El peor servicio que se puede hacer al sistema educativo es adoptar una actitud complaciente con los datos del estudio... porque revelan carencias $y$ lagunas importantes. Son datos severos y deben convertirse en un acicate para mejorar"

(Citado en El País, martes 10 de marzo de 1998, pág. 35).

Desde mi punto de vista lo sustancial de esa opinión es aplicable a lo que está ocurriendo con el desarrollo del programa de integración escolar de alumnos con necesidades educativas especiales. Esto es, que los indudables avances, tanto cuantitativos como cualitativos, que se han producido en estos últimos años respecto a la escolarización integrada de ese colectivo de alumnos, están generando una complacencia que difumina las múltiples carencias, dificultades y lagunas que todavía tiene dicho programa y con ello impidiendo, como señalaba acertadamente el Sr. Nasarre en el 
caso que le ocupaba, que tales carencias se perciban como un acicate, en este caso, para progresar hacia una auténtica educación de calidad para todos, sin exclusiones.

Pero desde la perspectiva y el conocimiento que me da el haber participado muy directamente en el desarrollo de ese programa, durante los años que trabajé para el Ministerio de Educación en el Centro Nacional de Recursos para la Educación Especial (CNREE) primero, y en el Centro de Desarrollo Curricular(CDC) después, pienso que para avanzar y mejorar en el proceso de integración escolar, se ha de cambiar no sólo esa actitud de complacencia a la que me he referido en primer lugar, sino también algunos de los elementos o decisiones que han venido configurando lo que podríamos llamar "el modelo de integración seguido por el MEC".

Para mi siempre ha sido muy esclarecedor ese refrán que dice que "el camino del infierno está empedrado de buenas intenciones". En efecto, muchas más veces de las que creemos, se toman decisiones que - sinceramente y con la mejor de las intenciones - , nos conducen, sin embargo, a destinos y resultados que no sólo no eran los esperados, sino que pueden ser además contrapuestos a la meta perseguida.

En mi opinión decisiones importantes relativas al "diseño" del programa de integración que el MEC adoptó en su momento y que se mantienen todavía, no sólo no están sirviendo para proporcionar una educación de calidad al alumnado con n.e.e., sino que están actuando en detrimento de ésta y haciendo un flaco servicio a la tarea de mejorar la educación para todos. Por esa razón en un segundo apartado de este artículo me detendré a analizar algunas de aquellas decisiones $y$, en particular, los siguientes aspectos:

1․ La opción por mantener, después de un más que razonable proceso de implantación una "doble red de centros" en lo que a la integración se refiere, y que está formada, por un lado, por aquellos "adscritos al programa" o que desarrollan esta tarea, y por otro el resto.

$2^{\circ}$. Los mecanismos que se están siguiendo para dotar de los recursos especiales materiales y humanos -que se necesitan para desarrollar la integración, lo cual está sirviendo, entre otras cosas, para reforzar, en lugar de minimizar como sería deseables, las prácticas de categorización del alumnado con n.e.e. que siguen practicándose, a veces a la más rancia usanza, y de espaldas a los avances que al respecto se están produciendo en ámbitos como el de la conceptualización del retraso mental ( AAMR, 1997).

Con todo, los cambios en esos parámetros serían también insuficientes si no se acompañaran de un decidida y generosa política en los ámbitos propios de lo que se ha venido en llamar "desarrollo curricular" (desarrollo de materiales y recursos didácticos, innovación e investigación educativa, documentación,...) y, muy en particular, en la formación permanente del profesorado. De ello depende que el profesorado, que en última instancia es el responsable de la mejora en la educación del alumnado con n.e.e., se encuentre con la motivación, la competencia y los apoyos necesarios para acometer el trabajo que se le pide , $y$ de forma que la meta prescrita se sienta como realmente alcanzable.

En este sentido, y con ello entraría en el tercer y último bloque de contenidos que pretendo revisar en este trabajo, creo necesario llamar la atención sobre la situación de abandono de la política de "desarrollo curricular" por parte de las actuales autori- 
dades educativas. Lo cual siendo grave para el conjunto de la población escolar, lo es más para la mejora de la escolarización del álumnado en desventaja, cual es el caso de aquellos con n.e.e..

En definitiva me parece importante señalar que la educación del alumnado con necesidades especiales no pasa por su mejor momento. Es más percibo que se encuentra en una fase de "retroceso", tanto por lo complejo de los siguientes pasos a dar -por ejemplo, la consolidación de la integración en la etapa de la educación secundaria obligatoria-, como por la falta de voluntad para enfrentarse a esa complejidad, y de convencimiento en el hecho de que la búsqueda de la equidad sea realmente el camino de la excelencia educativa para todos (Skrtic, 1991).

Aunque, como ya he señalado, mi experiencia más directa y profunda se refiere a lo que ha ocurrido y ocurre en el llamado, "territorio MEC" -y en ello me apoyaré básicamente para ejemplificar mis análisis y valoraciones-, creo que lo que se diga podría ser perfectamente aplicable en la mayoría de las Comunidades Autónomas que tienen desde hace tiempo competencias en estos temas. En cualquier caso, lo que más me mueve en esta ocasión a la hora de escribir este artículo no son los análisis comparados (que cada cual podrá realizar en su caso), cuanto que lo que pueda aportar sirva como elementos de reflexión y ayuda para muchos colegas que nos miran desde otros países, particularmente desde lberoamérica, queriendo aprender de nuestra experiencia. Y como todos sabemos, aprendemos mucho más de nuestros errores que de nuestros aciertos.

\section{Una meta y un camino.}

Del 7 al 10 de junio de 1994, más de 300 participantes, en representación de 92 gobiernos y 25 organizaciones internacionales se reunieron en Salamanca en la Conferencia Mundial que, con el título de "Necesidades Educativas Especiales. "Acceso y Calidad", permitió elaborar lo que desde entonces se conoce como Declaración de Salamanca y Marco de Acción sobre Necesidades Educativas Especiales (1994). La importancia de este encuentro fue muy grande en tanto en cuanto sirvió para señalar con claridad la meta que se ha de perseguir en la educación de este alumnado, haciéndolo con la fuerza del consenso mundial alcanzado. El trabajo de la Conferencia venía a ampliar además lo que Normas Uniformes de Naciones Unidas para la Igualdad de Oportunidades de las Personas con Discapacidad (Resolución 48/96, aprobada por la Asamblea General en su 480 reunión de 29/12/93), habían establecido a nivel general para el colectivo de personas con discapacidad.

En este sentido la "Declaración de Salamanca" (que debería ser un texto de estudio obligado y análisis detenido para todos aquellos realmente interesados en este tema), es suficientemente clara al respecto:

"3.(del Marco de Acción)

El principio rector de este Marco de Acción es que las escuelas deben acoger a todos los niños, independientemente de sus condiciones físicas, intelectuales, sociales, emocionales, lingüísticas u otras. .... Las escuelas tienen que encontrar la manera de educar con éxito a todos los niños, incluidos aquellos con discapacidades graves... Esta idea ha llevado al concepto de escuela inte- 
gradora... El mérito de estas escuelas no es sólo que sean capaces de dar una educación de calidad a todos los niños, con su creación se da un paso muy importante para intentar cambiar las actitudes de discriminación, para crear comunidades que acojan a todos y para conseguir sociedades integradoras"

\section{UNESCO (1994) Declaración de Salamanca y Marco de Acción, pág.6. Madrid: UNESCO/MEC}

Es obvio decir que la adopción de este principio no fue algo improvisado ni sin fundamento. Los propios trabajos preparatorios de la Conferencia y los múltiples y lúcidos análisis de expertos en política educativa (Fulcher, 1989; Ainscow, 1991; Wang, Reynolds y Walberg; 1995), han puesto de manifiesto repetidamente que los esquemas de pensamiento y las prácticas segregadoras seguidas respecto a la educación de los alumnos y alumnas con necesidades educativas especiales, no han servido para proporcionar a esas personas, llegado el momento, la "calidad de vida" (Verdugo, 1994.), a la que tienen derecho, ni han servido para mejorar los propios sistemas educativos.

Es obvio decir también que la meta ahora propuesta es de tanto calibre como pedir una transformación radical de la inmensa mayoría de los sistemas educativos vigentes; ¿es eso posible? Lo que todos los expertos señalan es que en un sistema de la complejidad del "sistema educativo", sólo parecen viables las innovaciones o reformas que parten de lo establecido y siguen un ritmo de cambio pausado o prudente (OCDE, 1996). Si tal es la situación, to que tenemos por delante respecto a la integración escolar, es un muy largo y complejo proceso, repleto de obstáculos, y "falsos caminos" que pueden hacer perder el norte al más pintado y, a la larga, dejar en muy poco las altas aspiraciones que la Declaración de Salamanca ha fijado.

En este contexto los "programas" de integración de alumnos con n.e.e., son, por lo tanto, un medio para acercarnos a esa meta que, no perdamos de vista, es la de proporcionar a todo el alumnado, sin discriminaciones ni exclusiones, una educación de calidad. Ese ha sido el camino seguido por la inmensa mayoría de los países de nuestro entorno, aunque con estrategias y resultados muy dispares ( Hegarty, 1993; OCDE, 1995).

No voy a repasar el conjunto de características y decisiones que han ido configurando el programa de integración del MEC durante estos años, tarea que he realizado ya en anteriores ocasiones (Echeita, 1991, 1992), y que el propio Ministerio de Educación hizo en su momento, junto con el último análisis evaluativo que se conoce hasta la fecha respecto a la situación de la integración escolar en su ámbito de competencias (MEC,1994). Como señalaba en la introducción, uno de mis objetivos es analizar algunas de las características del programa que, a mi juicio, nos alejan de la meta señalada y nos conducen, metafóricamente hablando, a un infierno no deseado.

$Y$ me parece muy importante hacer este tipo de análisis señalando, en primer lugar y aunque sea de forma telegráfica, los importantes "logros" alcanzados, entre los cuales no es el menor el propio hecho de que a estas alturas no se haya puesto en cuestión la continuidad de este aspecto de la política educativa, en un momento en el que lo tocante a la "atención a la diversidad" no tiene a sus mejores partidarios entre los responsables educativos del Ministerio de Educación y Cultura. 
Aunque en este sentido las valoraciones que podemos hacer son globales e imprecisas porque carecemos de evaluaciones rigurosas y representativas (lo cual es, por otra parte una de las grandes obstáculos que tiene en estos momento el programa de integración, pues se toman o dejar de tomar decisiones sin tener datos fiables en los que basarse), no cabe duda de que se pueden señalar los siguientes e importantes logros:

1. Es incuestionable que se ha producido un cambio significativo en las actitudes sociales hacia la integración escolar. Lo que hace $12 / 13$ años se veía como un planteamiento casi utópico o circunscrito a algunas experiencias muy limitadas, hoy se ha transformado en una actitud mayoritariamente positiva. Es difícil encontrar a quienes abiertamente cuestionan la importancia y necesidad de la integración escolar como pilar fundamental para conseguir la integración social y la equiparación de oportunidades de las personas con discapacidad ${ }^{1}$, (o de otros colectivos en desventaja).

2. Es un hecho que el programa se ha extendido hasta alcanzar un número de alumnos con n.e.e. escolarizados en centros ordinarios que alcanza, aproximadamente a $2 / 3$ del total de ese alumnado, al menos en el llamado "territorio MEC" (MEC, 1995). Aunque carecemos de datos evaluativos fiables respecto a lo que ha supuesto para ese alumnado la integración ${ }^{2}$ en términos de desarrollo y aprendizajes, hay motivos sobrados para pensar que, sin duda alguna, muchos de ellos están teniendo oportunidades y experiencias de aprendizaje muy positivas, que no hubieran alcanzado de estar escolarizados en centros específicos.

3. Si el número de alumnos integrados es importante, lo es porque el número de profesores implicados en esta tarea y el número de centros en los que se desarrolla también es importante. Detrás de esta iniciativa ya no están unos pocos "pioneros", sino una parte sustancial del colectivo de docentes que, sin menoscabo de los análisis críticos que se puedan hacer respecto a su trabajo en lo tocante a la respuesta educativa que ofrecen a este alumnado, son el principal baluarte de esta política junto con las familias de los alumnos con n.e.e. Estás están ahora mucho mejor organizadas y son muy conscientes de su papel, de sus derechos y de las metas que quieren alcanzar. (Basoco et al, 1997).

4. Por último, merece la pena señalar que, a pesar de las enormes dificultades, limitaciones y resistencias habidas en la etapa de la educación secundaria, la integración escolar se ha implantado en toda la enseñanza obligatoria, asegurando de esa forma que los alumnos con n.e.e. tengan acceso a una etapa decisiva para ellos y para sus iguales sin necesidades especiales a la hora de

1..Es cierto también que habría que matizar mucho el sentido y la organización de dicha integración escolar en el caso de colectivos como el del alumnado sordo profundo, situación sobre la que han llamado la atención tanto la Declaración de Salamanca como las Normas Uniformes.

2. Me parece muy importante volver a señalar que este tipo de investigación evaluativa debería tener carácter prioritario tanto para la Administración como para aquellos investigadores universitarios especializados en este ámbito educativo. 
configurar su identidad y su capacidad de inserción en la vida adulta y laboral (Fierro,1991)

Sin duda alguna se quedan en el tintero abundantes referencias de hechos y progresos que se podrían valorar con muy positivos; por ejemplo, el sustancial aumento de profesionales de la orientación educativa y psicopedagógica que, en su inicio, crecieron al socaire de la integración y que han supuesto un indudable punto de apoyo para el desarrollo del programa: la creación de recursos didácticos, y ayudas técnicas que se han puesto al servicio del profesorado o de los alumnos con n.e.e. directamente, o el desarrollo exponencial de trabajos y grupos de investigación sobre la amplia temática de la integración escolar( Arnaiz y P. De Haro, 1997.). No pretendo desmerecer nada de lo conseguido pero tampoco centrarme en ello.

Porque también es cierto que lo más importante y determinante a la hora de valorar los cambios que se han producido en el panorama de la atención educativa al alumnado con necesidades especiales, ha sido sacar adelante una ley como la LOGSE, que ha hecho de la "atención a la diversidad" uno de sus ejes y objetivos fundamentales y que ha establecido un marco normativo y unas condiciones a partir de las cuales es posible (aunque obviamente no suficiente) alcanzar el objetivo de una educación de calidad para dichos alumnos y alumnas. (Echeita, 1997)

Ahora bien, como he señalado desde el inicio, creo que hay estar extremadamente alertas y vigilantes de lo que ocurre, porque la complacencia con los logros, puede ser la peor compañera a la hora de enfrentarse a los muchos y complejos objetivos que quedan por cumplir.

En este sentido quiero recordar que, en mi opinión, estamos muy lejos de :

A. Que se perciba la integración del alumnado con n.e.e. (o de cualquier otra minoría en desventaja), como una estrategia privilegiada para que se asiente en las comunidades educativas la educación en valores, en particular "el valor del respeto y la apreciación de las diferencias" individuales, cualesquiera que estas sean.

B. Haber evitado que se vivencie esta tarea como propia solamente de algunos centros ("los centros de integración") y mayoritariamente públicos, de algunos profesores (de aquellos a los que "les toca en su clase" y sobre todo del "profesorado de apoyo"), y que es adecuada sólo para "algunos alumnos de entre los que tienen n.e.e." ("los alumnos de integración").

C. Estamos lejos de haber proporcionado al alumnado escolarizado en los centros de integración un nivel de enseñanza equiparable "en calidad" al que se ofrece al alumnado sin n.e.e. .

D. Estamos lejos de haber alcanzado en la etapa de la educación secundaria, lo alcanzado en la etapa de la educación primaria, si quiera en cuanto a número de alumnos integrados y atención recibida por estos.

E. De haber evitado que los alumnos con n.e.e. más graves y permanentes asociadas a discapacidades múltiples y complejas, no se vean más desatendidos en los centros de educación especial por efecto de que -"la integración"- ha acaparado la atención y los recursos. Aunque también es cierto que en algunas 
comunidades autónomas, como es el caso de Galicia, la mayoría de los alumnos con n.e.e. siguen en centros o aulas de educación especial (MEC, 1995).

F. De haber conseguido proporcionar a algunos colectivos específicos de alumnos con n.e.e.. como es el caso de los alumnos sordos profundos, una alternativa viable que conjugue la satisfacción de sus necesidades comunicativas con su participación en contextos escolares lo más normalizadores posibles (DíazEstébanez y Valamaseda, 1995).

Como señalaba más arriba los determinantes de esa situación son más que la mera complacencia con lo conseguido. En el apartado siguiente analizaré aquellos otros aspectos que apunté al inicio y que también me parecen centrales. Estos se sitúan unos en la esfera de las decisiones respecto al diseño del programa y otros en el plano de la responsabilidad individual de los docentes.

\section{Obstáculos "estructurales".}

\subsection{La "doble red de centros" en el ámbito de la integración escolar}

Parece razonable y sensato pensar que, dado que el número de alumnos con n.e.e. es reducido y que las inversiones en recursos humanos e infraestructuras son difíciles de generalizar, una opción posibilista es concentrar a dicho alumnado en determinados centros (los centros de integración) y hacer lo propio con los recursos humanos necesarios (profesorado de apoyo, logopedas, etc), y con las inversiones en ayudas técnicas y adaptaciones de espacios que también se precisan en algunos casos (por ejemplo en los centros de integración que escolarizan a alumnos con n.e.e. asociadas a una discapacidad motora).

Es indudable que esta estrategia, que es la seguida por el MEC, ha servido para conseguir en un tiempo relativamente breve (para lo que son los tiempos de cambio en educación), que el proceso de integración se esté desarrollando en todas las poblaciones cubriendo, por lo general, las demandas de escolarización previstas. Ahora bien, también ha servido para reforzar en el conjunto del profesorado y de las familias con hijos con y sin n.e.e. actitudes contrarias a la meta a largo plazo a la que se aspiraba.

En efecto no creo exagerar al afirmar que para buena parte de la comunidad educativa, la integración escolar, sigue siendo, fundamentalmente, una cuestión de "recursos" y de "especialistas". Actitud que, vaya por donde, le hace flaco servicio a "la reforma" en su objetivo de llevar al interior de todos los centros escolares en cualquier etapa educativa, el principio de atención a la diversidad, y en el de potenciar en el alumnado actitudes y valores relativos al respeto y valoración de las diferencias individuales. En definitiva, no estamos sino reforzando justamente lo que se quería superar ("la mejor forma de atender a la diversidad es que esta se concentre en algunos centros bien dotados de recursos y "especialistas" para atender adecuadamente a las especiales necesidades de ese alumnado"), y que no es otra cosa muy distinta del viejo esquema de la educación segregada en centros de educación especial. "El camino del infierno..." 
Si tenemos presente además que este programa ha sido escasamente asumido por los centros concertados y privados ${ }^{3}$, el efecto negativo de esta estrategia queda ampliado: "el sistema de educación pública es el que debe asumir la atención a la diversidad en lo que tiene que ver con los colectivos de alumnos en desventaja". Flaco servicio el que esta decisión hace a la "imagen" del sistema público de educación, en un momento en el que los aires "liberales" que nos rodean hacen todo lo posible para desacreditar lo público.

Soy de la opinión de que ese esquema resultó válido en su momento y muy particularmente en la fase de inicio del programa en la que era necesario mostrar que "la integración era posible". En esas fechas la concentración de esfuerzos y sinergías era imprescindible para vencer las resistencias de las comunidades educativas frente a la integración, que para entonces era una situación desconocida y casi utópica.

Pero hace tiempo que debería haberse superado, asegurando que todos los centros sostenidos con fondos públicos escolarizaran de manera equilibrada a los alumnos sin n.e.e. que naturalmente les correspondieran de acuerdo con los criterios establecidos para la escolarización del alumnado en general. Ciertamente así lo estableció la Ley Orgánica de la participación, la evaluación y el gobierno de los centros docentes, pero en los últimos tiempos parece que las leyes están para ser criticadas, pero no para ser acatadas. La "insumisión educativa" está al orden del día y no parece que a muchos les preocupe.

\subsection{Ratios de alumnos con n.e.e. y profesorado especialista.}

Parece razonable y sensato pensar que una forma segura de comprometer a la administración educativa con la dotación del profesorado de apoyo especializado que se precisa para una mejor educación de los alumnos con n.e.e. es establecer, con rango de norma de obligado cumplimiento, una relación que fije la dotación de dicho profesorado en función de un determinado número de alumnos con n.e.e. y de sus necesidades específicas. Eso es lo que se hizo con la Orden de 18 de Septiembre de 1990 por la que se establecen las proporciones de profesionales/alumnos en la atención educativa de alumnos con n.e.e. (BOE de 2 de octubre de 1990).

Con esa norma es cierto que se ha conseguido ampliar substancialmente el número de profesionales dedicados a la atención educativa del alumnado con n.e.e., ( y por ende la calidad de ésta), ${ }^{4}$ algo que podría haberse quedado en la esfera de las "buenas intenciones" si no se hubiera estructurado de esa forma.

Pero no es menos cierto también que ese esquema ha desencadenado dos conjuntos de efectos "perversos" que no hacen sino reforzar aspectos de los esquemas tradicionales de la educación especial que pretendíamos superar, a saber:

3. En el informe del MEC (1994), y referido al curso 1993/94, se indicaba que existían 2.088 centros públicos que escolarizaban alumnos con n.e.e. en E.Infantil y Primaria, frente a 123 centros concertados para las mismas etapas

4. En el informe del MEC (1994), se señala la cifra de 3.673 maestros especialistas en apoyo al programa de integración en las etapas de E. Infantil y Primaria. 
1. La tendencia inequívoca por parte del profesorado a "buscar" entre su alumnado a aquellos que satisfagan en número y criterio (categorización) lo establecido por la norma administrativa. Si para tener un profesor de apoyo en un centro ordinario es necesario que se tengan escolarizados en el centro a 9/12 alumnos "con deficiencia psíquica" (criterio establecido en la Orden citada), que a nadie le quepa la menor duda que "los centros" terminarán encontrando el número de alumnos requeridos con esa deficiencia para no perder "el recurso".

2. La inequívoca tendencia de la administración a responder a las demandas de recursos por parte de los centros, que se perciben como "abusivas o injustificadas", por la vía de hacer más restrictivo el criterio de dotación de los mismos.

Y así ha ocurrido que al tiempo que se predicaba como uno de los grandes avances en la conceptualización de las necesidades especiales, el carácter interactivo y por lo tanto relativo de la misma (MEC, 1992), y el que este planteamiento permitía superar los efectos negativos que las etiquetas diagnosticas habían tenido en las expectativas que volcamos sobre éste alumnado centrando nuestra atención sobre las necesidades educativas y los apoyos que este alumnado precisara ${ }^{5}$, a la hora de dotar con profesorado especializado a los centros, el número de estos se hace depender de que existan tantos o cuantos alumnos categorizados con las mismas etiquetas que decíamos querer superar (deficiencia mental, deficiencia sensorial, "motóricos", etc)..

Soy de la opinión que han de buscarse nuevos esquemas para la necesaria dotación y financiación de los recursos extraordinarios que se necesitan para la escolarización integrada de los alumnos con n.e.e., como los que se han puesto en práctica en el distrito 12 de la provincia de New Brunswick, Canadá (Porter,1995). Allí su Ministerio de Educación financia la "educación especial" o mejor dicho "el servicio de estudiantes" que es como se denomina en ese distrito, proporcionando a los distritos (y no a los centros directamente), una asignación por alumno para gastar en "servicios de estudiantes", basada en la población de estudiantes total del distrito. Por ejemplo, el suyo recibe una asignación de $300 \$$ por alumno; como tiene 5000 alumnos eso da lugar a un presupuesto de 1'5 millones de $\$$ para gastar en servicios especiales.

Este esquema de financiación tiene, comparativamente con el que nosotros aplicamos, importantes ventajas:

1. Elimina la necesidad de justificar la financiación basándose en los tipos y grados de discapacidad de los alumnos

2. Traslada su foco de las condiciones de discapacidadd a los servicios de apoyo para los profesores y los alumno con n.e.e.

3. No se refuerza ni anima a que se designen alumnos con discapacidad. Asume que cada distrito y cada escuela necesitará un cierto nivel de servicios de apoyo simplemente porque las escuelas atienden a una población heterogénea de alumnos, porque todas asumen "la atención a la diversidad".

5. En plena concordancia con lo que hoy se pretende a través de la nueva conceptualización del "retraso mentai" (AARM, 1997) 
4. Estimula la responsabilidad y la optima rentabilización de los recursos propios, tanto a nivel de distrito como en cada centro escolar, reforzando con ello el papel de una dirección escolar con liderazgo

5. El hecho - muy importante - de que la financiación se sitúe a nivel de distrito permite una flexibilidad y una posibilidad de ajuste en la adjudicación de recursos, en función de las necesidades individuales de cada escuela.

No estoy proponiendo trasladar sin más este esquema a nuestro contexto, algo a todas luces imposible pues el mismo guarda relación con otros muchos aspectos del sistema educativo canadiense que no son aplicables a nuestra sistema escolar por más que quisiéramos. Lo que abogo es por repensar urgentemente las prácticas de financiación que estamos siguiendo pues, una vez más, "El camino del infierno ..."

\section{Una política de desarrollo curricular que brilla por su ausencia.}

¿A alguien del mundo educativo se le ocurre una tarea más compleja para el profesorado que la de tratar de ofrecer a un conjunto de alumnos diversos - algunos de ellos tal vez con necesidades especiales -, en una etapa como la de la E.S.O., por ejemplo, una enseñanza de calidad en un mismo contexto educativo? Desarrollar en todas sus dimensiones el principio educativo de atención a la diversidad (Coll, 1994) sólo es posible si se acompaña, entre otras medidas, de una clara, sostenida y firme política de desarrollo curricular. La LOGSE así lo entendió y señaló en su Título cuarto los factores más importantes de cuya confluencia cabía esperar un avance sustancial en una mejor educación para todos. Entre estos habría que destacar los que tienen que ver con la innovación y la orientación educativa, con la formación permanente del profesorado, con la elaboración y difusión de materiales curriculares, con la información y el asesoramiento o con el acceso a recursos didácticos de utilidad.

A ese empeño global dedicó el anterior Ministerio de Educación un sin par esfuerzo económico y humano que incluso sería difícil resumir. Entre el mismo no fue el menor el dedicado en particular al ámbito de la educación de los alumnos con necesidades educativas especiales y creo que los avances señalados anteriormente no hubieran sido del calibre indicado sin la inversión realizada en este sentido. La creación del Centro Nacional de Recursos para la Educación Especial (CNREE), que funcionó entre 1985 y 1995 y la posterior del Centro de Desarrollo Curricular (CDC) hasta su desaparición en 1996, fueron decisiones que sirvieron para concretar y poner en manos de las comunidades educativas recursos necesarios para la nueva tarea que se les demandaba y, sobre todo, un punto de referencia y un foro de análisis para tratar los problemas del desarrollo de la política de integración escolar..

Sería justo y necesario que se pudiera valorar con equidad y perspectiva el trabajo realizado por esos centros ( junto con el de la red de CPRs.), con relación a su contribución en la mejora de la atención a la diversidad en general y en el ámbito de las necesidades especiales en particular. No creo ser la persona adecuada para ello, ni busco "ensalzar lo pasado", pero la realidad hoy es que las políticas de desarrollo curricular brillan por su ausencia o debilidad. La desaparición "de facto" del Centro 
de Desarollo Curricular, (CDC) -cuyas funciones nominales asume sólo en teoría el actual Centro de Investigación y Documentación Educativa (CIDE); la descapitalización humana del Programa de Nuevas Tecnologías para la Información y la Comunicación (PNTIC), (en lo tocante al servicio que impulsaba e desarrollo de las nuevas tecnologías en el ámbito de las n.e.e.), junto con la lánguida subsistencia de los CPRs, se deja sentir día a día en los centros escolares y por los servicios de apoyo y orientación psicopedagógica, justo cuando era necesario, ante la complejidad de las tareas por desarrollar, un mayor esfuerzo y empeño para ayudar al profesorado a enfrentar las dificultades de la atención a la diversidad con la perspectiva y el talante de un desafió personal y profesional.

\section{Responsabilidades y actitudes frente a la integración escolar.}

Hasta aquí he querido poner de manifiesto que existen actitudes sociales y condiciones organizativas que están condicionando de forma negativa, a mi modo de ver, el desarrollo de la política de integración escolar.

Con ser todas ellas importantes, en último término, no eximen al profesorado, por completo, de su responsabilidad ante la tarea de atender adecuadamente a los alumnos con necesidades educativas especiales. Con demasiada frecuencia y con el argumento de lo complicado de la tarea o de la ausencia de ayudas, he visto a muchos profesores hacer dejación de las funciones y tareas que las normativa establece para educar en condiciones de igualdad al alumnado con necesidades especiales. Aquel refrán de "a Dios rogando y con el mazo dando" sería de justa aplicación en lo que ahora nos concierne. Exijamos la mejora de las condiciones laborales, el aumento de ayudas técnicas y personales para poder responder a la complejidad de nuestra aulas, y los cambios "estructurales" que sean pertinentes, pero que ello no sirva de escusa o argumento para la parálisis o el descuido de la educación de este alumnado.

Alguien, muy acertadamente, dijo en una ocasión que la política educativa, no es sólo lo que hace la Administración y lo que figura en las leyes y normas; también la hace cada profesor o profesora cuando entra en su clase y cierra tras de si la puerta de su aula.

En definitiva, con todo lo señalado hasta aquí, creo que no corren "buenos tiempos para la lírica" en lo referido a la educación del alumnado con necesidades especiales, y lo peor, en el fondo, no son las actitudes, errores $y$ ausencias señaladas, cuanto una especie de actitud de "inevitable derrotada anunciada" frente a la política ministerial que parece inmovilizar a la comunidad educativa, a las familias de los alumnos con necesidades especiales, a la comunidad universitaria $y$, en particular, a muchos profesionales que en otro tiempo fueron "azote de prácticas y conciencias".

En la conferencia de inaguración de la Conferencia Mundial de Salamanca, Mayor Zaragoza recordaba lo que le había dicho una persona discapacitada:

"Las actitudes son rnás importantes que los hechos, que las circunstancias, que los fracasos o los éxitos. Las actitudes crean o destruyen una amistad, un hogar. 
Pero..., cada día podemos decidir la actitud que vamos a adoptar. La vida es un diez por ciento lo que sucede y un noventa por ciento cómo reaccionamos ante ello. Somos responsables de nuestras actitudes."

UNESCO (1994) Informe Final. Conferencia sobre Necesidades Educativas Especiales. Acceso y Calidad. Madrid: UNESCO/MEC

Somos responsables de nuestras actitudes. Lo que sucede en estos momentos es que las tareas que tenemos por delante respecto a la educación del alumnado con necesidades especiales son las más difíciles y costosas, las que va a requerir de nosotros mayor creatividad e ingenio y las que va a poner a prueba la voluntad política y la firmeza de las convicciones de quienes con relativa facilidad asumen las grandes declaraciones de principios. De nuestra actitud, de la de aquellos que creemos en la igualdad y en la solidaridad, dependerá, en efecto, que estemos frente a una "derrota anunciada", o un paso más cerca de ver una educación de calidad para todos, sin exclusiones.

\section{Referencias}

AAMR (1997) Retraso Mental. Definición, clasificación y sistemas de apoyo. Madrid: Alianza Psicología

AINSCOW, M. (1991) Effective Schools for Al/ Londres: David Fullan

ARNAIZ, P. y P del HARO,R. (Eds) (1997) 10 años de integración en España. Análisis de la realidad y perspectivas de futuro. Murcia. Servicio de Publicaciones de la Universidad de Murcia

BASOCO, J.L. et al (1997) "La persona con retraso mental y sus necesidades: mejora de su calidad de vida" Siglo Cero, 169, 28(1), 5-18

COLL,C. (1994) "El tractament de la diversitat a la LOGSE" Espais Didactics, 7, 14-22

DÍAZ-ESTÉBANEZ, E. y VALMASEDA, M. (1995) "En el camino hacia una educación de calidad para los alumnos y alumnas sordos "Infancia y Aprendizaje, 69-70, 45-60

ECHEITA, G. (1991). "El Programa de Integración del MEC". Cuadernos de Pedagogía, $191,72-75$.

ECHEITA, G. (1992). "La integración escolar a debate". "Aula de Innovación Educativa", 6, 73-77.

ECHEITA, G. (Coordinador) (1997) Atenció a la diversitat i necessitats educatives especials. Barcelona: Universitat Oberta de Catalunya

FIERRO, A. (1991). "Las necesidades educativas especiales en la Reforma Educativa: el horizonte de la Secundaria". Siglo Cero, 135, 12-22.

FULCHER, G. (1989).Disabling Policies. Lewer: Falmer Press.

HEGARTY, S. (1993) "Reviewing the literature on integration" European Journal of Special Educational Needs, 8(3), 194-200

M.E.C. (1994) La educación especial en el marco de la LOGSE Madrid: Secretaria de Estado de Educación, Centro de Publicaciones, M.E.C.

M.E.C. (1992) "Adaptaciones Curriculares" EN Materiales de Apoyo para la Reforma Educativa. Primaria(Cajas Rojas). Madrid: M.E.C. Centro de Publicaciones

M.E.C. (1995)El sistema educativo español. Madrid: CIDE, Centro de Publicaciones M.E.C. 
SKIRTIC, T.M. (1991) "Students with special educational needs: Artifacts of the traditional curriculum" EN M. Ainscow (Ed.)(1991) Effective Scholls For All Londres: Fulton

OCDE (1995).Integrating students with special needs into mainstrean Schools. París: OCDE.

OCDE (1996) Teacchers and Curriculum Reform in Basic Schooling. "Systemic Reform and Lessons for Policy". París: CERI (Documento de Trabajo no publicado del proyecto de igual título)

PORTER, G. (1995) "Organización de la escuela: garantizar el acceso y la calidad a través de la inclusión "Prospects, 25(2), 299-310

UNESCO (1994).Informe Final Conferencia Mundial sobre Necesidades Educativa Especiales: Acceso y Calidad. Marco de Acción y Declaración de Salamanca. París: UNESCO/MEC.

VERDUGO, M.A. (1994) "El papel de la Psicología de la Rehabilitación en la integración de personas con Discapacidad y en el logro de calidad de vida", Siglo Cero, 25(6), 33-42

WANG, M.G.; REYNOLDS, M.G. y WALBERG, M. (1995).Handbook of Special and Remedial Education. Research and Practice. Second Edition Oxford: Pergamon 
